\title{
Acknowledgement to Referees
}

(c) Springer Nature Switzerland AG 2018

\section{Dear Reader}

As we reach the final issue of Drugs for 2018, we wish to reflect on another successful year's achievements, and to thank all those who have contributed their time and effort to guarantee the high quality of our content.

2018 was a successful year for Drugs. Over 150 articles have been published, and as of the end of October, 31 of these have been downloaded from SpringerLink over 1000 times. Topics covered by these popular articles included switching reference medicines to biosimilars, tiotropium use in children and adolescents, management of inherited von Willebrand disease, the use of metformin in prediabetes and diabetes prevention, evaluations of new antibiotics, and drugs in development for spinal muscular atrophy, viral hepatitis and malaria.

Social media sharing of information is becoming more important and is another metric with which to measure the reach of articles. Again this year, several articles published in Drugs scored over 20 on the Altmetric system. The quality of articles published in the journal is also reflected in its most recent impact factor of 4.69.

Other journals in the Adis Premier Journals Portfolio (http://www.springer.com/gp/adis) continue to prosper with substantial increases in usage year on year. In addition, this year has seen Applied Health Economics and Health Policy receive its first impact factor.

We thank the authors who have contributed articles to Drugs over the course of 2018. The skill and dedication of all authors are critical to the continued publication of the journal. The quality of published articles is also testament to the significant efforts of the peer reviewers, whose commitment ensures that the journal's content is held to the highest possible standard. We would like to thank the following individuals who acted as reviewers for Drugs in the last 12 months:

\author{
Carlos Abud-Mendoza, Mexico \\ Piotr Adamski, Poland \\ Anurag Kishor Agrawal, USA \\ Mehmet Akman, Turkey \\ Praveen Akuthota, USA \\ Rieke Alten, Germany \\ John Anderson, UK \\ Richard Anderson, UK \\ Karl-Eric Andersson, USA \\ Desiree Anthony, Australia \\ Camila C. H. Aquino, Canada \\ Louis J. Aronne, USA \\ Antonio Ascione, Italy \\ Sait Ashina, USA \\ Rabea Asleh, USA \\ Victoria Atkinson, Australia \\ Luis Bahamondes, Brazil \\ Osnat Bairey, Israel \\ Gowrie Balasubramaniam, UK \\ Herbert S. B. Baraf, USA \\ Janna C. Beavers, USA
}

\author{
Vikhyat Bebarta, USA \\ Stefano Bellosta, Italy \\ Lars Bendtsen, Denmark \\ Laura Benítez-Gutiérrez, Spain \\ Carola Berking, Germany \\ Sonia Berrih-Aknin, France \\ Gaetano Bertino, Italy \\ O. Giles Best, Australia \\ Jeffrey R. Boris, USA \\ Ray Borrow, UK \\ Helen W. Boucher, USA \\ Melissa Bowerman, UK \\ Adam P. Bress, USA \\ Francesco Brigo, Italy \\ Kirsten M. Boughan, USA \\ Adam M. Brufsky, USA \\ Catharine C. Bulik, USA \\ David M. Burger, The Netherlands \\ Jane Buxton, Canada \\ Tommaso Cai, Italy \\ Natalie Callander, USA
}


Luis H. Camacho, USA

Bangwei Cao, China

Teofila Caplanusi, Belgium

Mariana Castanheira, USA

Dario Cattaneo, Italy

Zeynep Kendi Celebi, Turkey

Claudio Cerchione, Italy

Jeremy Chataway, UK

Irini Chatziralli, Greece

Meera Chitlur, USA

Francesco Ciccia, Italy

Laura C. Coates, UK

Alexander T. Cohen, UK

E. Ross Colgate, USA

Brenda Cooper, USA

Giorgio Cruccu, Italy

Lloyd E. Damon, USA

Chintan V. Dave, USA

Michele De Laurentiis, Italy

Prakash C. Deedwania, USA

Ulrich Desselberger, UK

Aikaterini Detoraki, Italy

Giovan G. Di Costanzo, Italy

Michael P. Diamond, USA

Hans-Christoph Diener, Germany

George Dimitriadis, Greece

Luc Y. Dirix, Belgium

Paul P. Dobesh, USA

Fernando Docobo-Perez, Spain

Zedong Du, China

Stephan Duparc, Switzerland

Charmane I. Eastman, USA

Robert Egerman, USA

Michele F. Eisenga, The Netherlands

Akram Y. Elgendy, USA

Islam Y. Elgendy, USA

Cathy Eng, USA

Georg Ertl, Germany

Luis R. Espinoza, USA

Hasan Fadlallah, USA

Haiming Fang, China

Michelle A. Farrar, Australia

Michael Farrell, Australia

Claudio Feliciani, Italy

Maria Fernandez Cortizo, Spain

Kate Fife, UK

David Fitchett, Canada

Masahide Fukudo, Japan

Jan Gaertner, Switzerland

Maurizio Gallieni, Italy

Jing Gao, China

Bradley J. Gardiner, USA

Emily M. Garland, USA
Kathy Gately, Ireland

Helen Giamarellou, Greece

Maria A. Giamberardino, Italy

Riccardo Giampieri, Italy

Peter Gibbs, Australia

Antonietta Gigante, Italy

Nils Erik Gilhus, Norway

Eilish Gilvarry, UK

Catherine Godfrey, USA

Ricardo Gómez-Huelgas, Spain

Priscila H. Barcellos Goncalves, USA

S. Gonnelli, Italy

Isidoro Gonzalez-Alvarez, Spain

Garry G. Graham, Australia

Megan E. Griffith, UK

David J. Gross, Israel

Franjo Grotenhermen, Germany

Luigina Guasti, Italy

Sonia Guedan, Spain

Anupam Datta Gupta, Australia

Kieran Halloran, Canada

Guy Hans, Belgium

Nordin Hanssen, The Netherlands

Craig T. Hartrick, USA

Takashi Hashimoto, Japan

Don Hayes, USA

Hiddo J. Lambers Heerspink, The Netherlands

Philip S. Helliwell, UK

Steen W. Henneberg, Denmark

Ignacio Herrero, Spain

Cecilia J. Hillard, USA

Rosario D. Crespo Hirata, Brazil

Swapnil Hiremath, Canada

Jian-Guo Hong, China

Dieter Hörsch, Germany

Tomoya Hoshi, Japan

Takahiro Inoue, Japan

Demetris Ioannides, Greece

Michael G. Ison, USA

Maartje S. Jacobs, The Netherlands

Heidi Janssen, Australia

Richard J. Johnson, USA

Marcel F. Jonkman, The Netherlands

Elmar A. Joura, Austria

Fabrice Journe, Belgium

Wojciech Jurczak, Poland

Tim P. Jürgens, Germany

Kevin Kalinsky, USA

Sang-Moo Kang, USA

Stylianos Karatapanis, Greece

Pashtoon Murtaza Kasi, USA

James S. Khan, Canada

Hyoun-Ah Kim, Republic of Korea 
Zaneta Kimber-Trojnar, Poland

Sarah R. Kingsbury, UK

Manish Kohli, USA

Anthony L. Kovac, USA

Florian Krammer, USA

Peter Kreiner, USA

Günther Krumpl, Austria

Ragini Kudchadkar, USA

H. Kuo, Taiwan

Pekka Kurki, Finland

Joseph L. Kuti, USA

Nebojsa Lalic, Serbia

Angela Lamarca, UK

Stefan Lauer-Riffard, Germany

Dean Lee, USA

Nelson Lee, China

Joel Leong, Singapore

Ying Ying Leung, Singapore

Michael Levy, USA

Timothy C. M. Li, China

Ajit Limaye, USA

Chia-Chi Lin, Taiwan

Sofia Lionaki, Greece

Josep M. Llibre, Spain

Philipp P. Koren Lobmaier, Norway

Francesco Locatelli, Italy

Maria A. Lopez-Olivo, USA

Rutger Maas, The Netherlands

Franco Maggiolo, Italy

Johnny Mahlangu, South Africa

Fabio Malberti, Italy

Una Martin, UK

Nicolas Martinez-Majander, Finland

Juan Martin-Liberal, Spain

Alan Mast, USA

Alexandre Matet, Switzerland

Ashish J. Mathew, India

Roy O. Mathew, USA

Yoshihiro Y. Matsukawa, Japan

Barbara Melosky, Canada

Cristina M. C. Meriggiola, Italy

Giovanni Battista Migliori, Italy

Moshe Mittelman, Israel

Mario-Luca Morieri, USA

Ryuichi R. Morishita, Japan

Bart Morlion, Belgium

Sergey M. Motov, USA

Thomas Müller, Germany

Kirsten Muller-Vahl, Germany

Kenneth A. Myers, Australia

Robert T. Naismith, USA

Hironori Nakagami, Japan

Bimota Nambam, USA
Aziz Nazha, USA

Mark Nelson, UK

Sarah Nevitt, UK

David P. Nicolau, USA

Naoyuki Nogami, Japan

Patrice Nordmann, Switzerland

Michael T. Nurmohamed, The Netherlands

Meg Oberle, USA

Nik Onufrak, USA

Ken Op de Beeck, Belgium

Anthony D. Ormerod, UK

Folashade Otegbeye, USA

Michael Overman, USA

Eugenia Oviedo-Joekes, Canada

Chetan P. Padke, Canada

Channing Paller, USA

Hardev Pandha, UK

Hugh M. Paterson, UK

Girolamo Pelaia, Italy

M.A. Pinna, Spain

Raffaella Pisapia, Italy

Lisa A. Porter, Canada

Martin Prázný, Czech Republic

Thomas Prebet, USA

Lluís Puig, Spain

Livia Puljak, Croatia

Henry A. Punzi, USA

Mariusz, J. Puszczewicz, Poland

Jukka Putaala, Finland

Robert B. Raffa, USA

Margaret Ragni, USA

C. Venkata S. Ram, USA

Abdul Qayyum Rana, Canada

Lori Reisner, USA

Christophe Richez, France

Lorenza Rimassa, Italy

Alan L. Robin, USA

Keith A. Rodvold, USA

Simon D. Roger, Australia

Jane Rogers, USA

Antonio A. Rossi, Italy

Euthalia Roussou, UK

Abdulrahim Rouzi, Saudi Arabia

Giandomenico Roviello, Italy

Christopher M. Rubino, USA

Mohammed Ruzieh, USA

Rainer Sabatowski, Germany

Emilio Sacco, Italy

Helio S. Sader, USA

Andrea Salonia, Italy

Álvaro Sánchez-Larsen, Spain

David R. Saxon, USA

Raffaele Scarpa, Italy 
Desmond A. Schatz, USA

André J. L. Scheen, Belgium

Amand F. Schmidt, UK

Adrian Schreiber, Germany

Stephan A. Schug, Australia

Roy Schwartz, UK

Nicola Scichilone, Italy

Eva Segelov, Australia

Michael G. Serpell, UK

Dennis Shanks, Australia

Catherine M. Turner Sherwin, USA

Kohei Shitara, Japan

Alpana P. Shukla, USA

Kenneth J. Simpson, UK

Rishi P. Singh, USA

Petros Skapinakis, Greece

Francois Spertini, Switzerland

Kevin Spring, Australia

Gitanjali Srivastava, USA

Rudolf E. Stauber, Austria

Jürgen Stein, Germany

Yu Sunakawa, Japan

Atsushi Tanaka, Japan

Thomas Tängdén, Sweden

Kemal Tekin, Turkey

Ursula Theuretzbacher, Austria

Carol B. Toris, USA

Christopher J. Twelves, UK

Muhammad Shariq Usman, Pakistan

Kimiaki Utsugisawa, Japan

Michael Van Ameringen, Canada

David van Duin, USA
Luc F. Van Gaal, Belgium

Frederik H. Verbrugge, Belgium

Eleni Vergadi, Greece

Alex Vorsters, Belgium

Alpo Vuorio, Finland

Adrian Wagg, Canada

Carsten A. Wagner, Switzerland

Laura H. Waite, USA

Zen'ichiro Wajima, Japan

Stephanie Weibel, Germany

Martin Weickert, UK

Niels Weinhold, USA

Mark Wenink, The Netherlands

Victoria P. Werth, USA

Sonja Werwitzke, Germany

Rene Westhovens, Belgium

Elaine C. Wirrell, USA

Christina Wu, USA

Lei-Bo Xu, China

Junichi Yamaguchi, Japan

Yoshiaki Yamaguchi, Japan

Tetsuya Yamamoto, Japan

Ian Yang, Australia

Jilong Yang, China

Harry H. Yoon, USA

Giuseppe Zagotto, Italy

Joanna M. Zakrzewska, UK

Marty Zamora, USA

Milos Žarković, Serbia

Fei Zhou, China

Hong Zhu, China

In addition, we would like to thank the members of the journal's Honorary Editorial Board, who have acted as peer reviewers and authors, and have provided guidance on journal content, policy and processes.

We do hope that you have found the articles published throughout the year in Drugs to be interesting and informative. The editorial schedule for 2019 is well under way, and the Section Editors and I are looking forward to bringing you many high-quality and authoritative articles over the coming year.

With best wishes

Dene C. Peters

Editor, Drugs 